\title{
Simultaneous VLBI/GMRT/RXTE observation of SS433
}

\author{
S. K. Chakrabarti ${ }^{1,2}, \mathrm{~S} . \mathrm{Pal}^{2}$, and A. Nandi ${ }^{2}$ \\ 1 S.N. Bose National Centre for Basic Sciences, JD Block, Salt Lake, Sector III, Kolkata 700098, India \\ e-mail: chakraba@bose.res.in \\ 2 Centre for Space Physics, Chalantika 43, Garia Station Rd. Garia, Kolkata 700084, India \\ e-mail: anuj@csp.res.in
}

Received 10 December 2005 / Accepted 10 March 2006

ABSTRACT

\begin{abstract}
Aims. Our aim in this paper is to discover the spectral characteristics of the enigmatic compact object SS433 during a very rare occasion when the blue jet was pointing towards us (the precessional phase $\psi=0$ ) and when the orbital phase $\phi$ of its companion was nearly zero (inferior conjunction) and compare this with the result obtained when $\phi \sim 0.5$ (superior conjunction).

Methods. We employed the Giant Meter Radio Telescope (GMRT) for the photometric observation at $1280 \mathrm{MHz}$, European VLBI Network (EVN) for photometry and imaging at $4990 \mathrm{MHz}$, and the Rossi X-ray Timing Explorer (RXTE) for photometry and spectrum at $3-25 \mathrm{keV}$.

Results. At this opportune moment, the companion totally blocked the disk and the base of the jet, the X-ray intensity was reduced by $20 \%$ with respect to the previous day, and the intensity went up by about $65 \%$ on the next day. The X-ray intensity is about onethird compared to RXTE results obtained during the superior conjunction on 13 March 2004. By taking the difference in the spectra, we obtained the spectrum of the system core including the contribution from any accretion shock that may have occurred during interaction between the accreting stream and the disk. The EVN observation clearly showed the separation of a radio blob, so using an adiabatic expansion model we estimate when the blob might have been separated.

Conclusions. We see a clear dependence of X-ray intensity emitted from the base of the jet on the photon energy. We find that the core is much brighter in hard X-rays. The conjunction seems to have had no effect on the radio data.
\end{abstract}

Key words. accretion, accretion disks - stars: binaries: eclipsing - stars: winds, outflows - radio continuum: stars - X-rays: stars methods: observational

\section{Introduction}

A large number of multiwavelength campaigns on SS433 (e.g., Neizvestnyj et al. 1980; Ciatti et al. 1981; Seaquist et al. 1982; Vermeulen et al. 1993; Band \& Gordon 1989; and Kotani et al. 1999; Revnivtsev et al. 2004; Chakrabarti et al. 2005) have been conducted so far and they have yielded very important results on many detailed characteristics of this enigmatic object. However, because of very large extinction, it has become impossible to decipher the true nature of this compact object and its companion. It is still not clear what causes the precession and nutation of the disk and the blobby but continuous jet that comes out of it at a nearly constant speed of 0.26 times the velocity of light. Because of these difficulties, one has to plan observations of the object for when it is in a special configuration at which some of the effects can be ignored while others are enhanced.

In this work, we present the results of SS433 in a very special time slot, when the blue-shifted jet is pointing directly towards us $(\psi=0)$, while the companion is directly blocking the disk and the base of the jet ( $\phi=0$, inferior conjunction). This opportune moment happened on 24 August 2004, at 7:12 UT when the precessional phase $\psi$ and the orbital phase $\phi$ of SS433 were nearly zero. Our observation is around this time frame from August 23 till August 28, 2004. We present results from the Giant Meter Radio Telescope (GMRT) for a $1.28 \mathrm{GHz}$ observation, the European VLBI Network (EVN) for a $4.99 \mathrm{GHz}$ observation, and the RXTE satellite for a 3-30 keV observation. Some preliminary analysis of our results was presented in Paragi et al. (2005). We also present the spectrum at the superior conjunction $(\phi=0.5)$ that occurred on March 13, 2004, while the jet was pointing towards us $(\psi=0)$ and compare it with the one taken at the inferior conjunction, thereby obtaining the spectrum of the disk and the core of the jet.

In the next section, we briefly describe of the instruments used in our campaign and in Sect. 3, we present the major results and our interpretations of these results. Finally, in Sect. 4, we make concluding remarks.

\section{Instruments used in the multiwavelength campaign}

The observations of SS433 by EVN are organised at $4.99 \mathrm{GHz}$ on 23, 25, and 27 August 2004, outside the EVN observing sessions. Participating telescopes were Urumqi, Torun, Onsala, Noto, Westerbork, Effelsberg, Shanghai, and Medicina (5 of these telescopes at any single epoch). The recording rate was $256 \mathrm{Mbit} / \mathrm{s}$, which resulted in $4 \times 8 \mathrm{MHz}$ channels in both left and right circular polarization. SS433 was observed for $4 \mathrm{~h}$ per epoch, and the data were correlated at JIVE. The NRAO AIPS package was used for post-processing (e.g. Diamond 1995). Imaging was carried out in Difmap (Shepherd 1997). The rms noise levels achieved were 50-100 microJy/beam, about what was expected with both our limited array and the short observing time available.

The GMRT observations were made at $1280 \mathrm{MHz}$ with a $16 \mathrm{MHz}$ bandwidth on $23-25$ and 28 August 2004. On the 28 th, all 30 antennas were working, while on the other days 


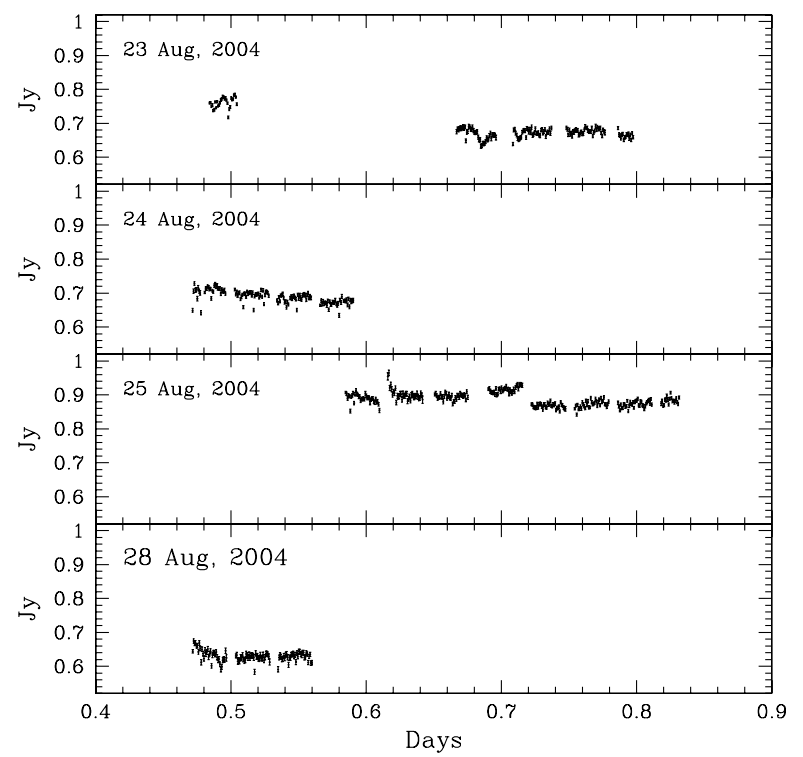

Fig. 1. GMRT lightcurves of SS433 showing considerable variation ranging from $\sim 0.6 \mathrm{Jy}$ on the 28 th to $\sim 0.9 \mathrm{Jy}$ on the 25 th. Lack of a correlation between the emitted flux with the conjunction indicates that most of the radio emission is taking place well outside the eclipsed region.

29 antennas received satisfactory signals. On each day several hours of data were taken and then properly flagged, calibrated, bandpassed, channel-averaged, back-ground subtracted, and self-calibrated using AIPS software package. 3C 286 was used as the flux calibrator on all the days, but on the 23rd and 24th, 3C 48 was also used as a flux calibrator. Each set of data was binned for $16 \mathrm{~s}$.

The RXTE observations were made during 23-25 August 2004 and a total of about two hours of data was collected. These were then analysed using the standard FTOOLS/XSPEC packages. Detailed methods of GMRT and RXTE data reduction for SS433 are already in Chakrabarti et al. (2005), and so are not repeated here.

\section{Results and interpretation}

In Fig. 1, we show the GMRT lightcurves of SS433. The actual data points with error-bars are shown. The energy flux density showed considerable variation in these days, ranging from $\sim 0.6 \mathrm{Jy}$ on the 28 th to $\sim 0.9 \mathrm{Jy}$ on the $25 \mathrm{th}$. But this seems to have no relation with the inferior conjunction that took place on 24 August 2004. This is expected as the radio emitting region $\left(\sim 10^{15} \mathrm{~cm}\right)$ is well outside the region blocked by the companion.

In Fig. 2, we show the light curves of the X-ray observation by RXTE. We clearly see a decline in the count rate on 24 August by a significant amount. The average count rates on 23,24 , and 25 August were $\sim 46, \sim 35$, and $\sim 52$, respectively. There were micro-variabilities in time scale of minutes on all these days as seen in previous observations (Chakrabarti et al. 2003).

In Fig. 3, we plot the energy flux densities from all the three instruments as a function of the day. We took mean values on each day. The point of time at which the inferior conjunction occurs is shown by an arrow. The shallow X-ray dip shows that not all the X-rays are blocked by the companion. In comparison to the previous result of March 2004 (Nandi et al. 2005), which reported the flux at the superior conjunction, we note that the total flux in this case is only about $40 \%$ (see Table 1 ). This

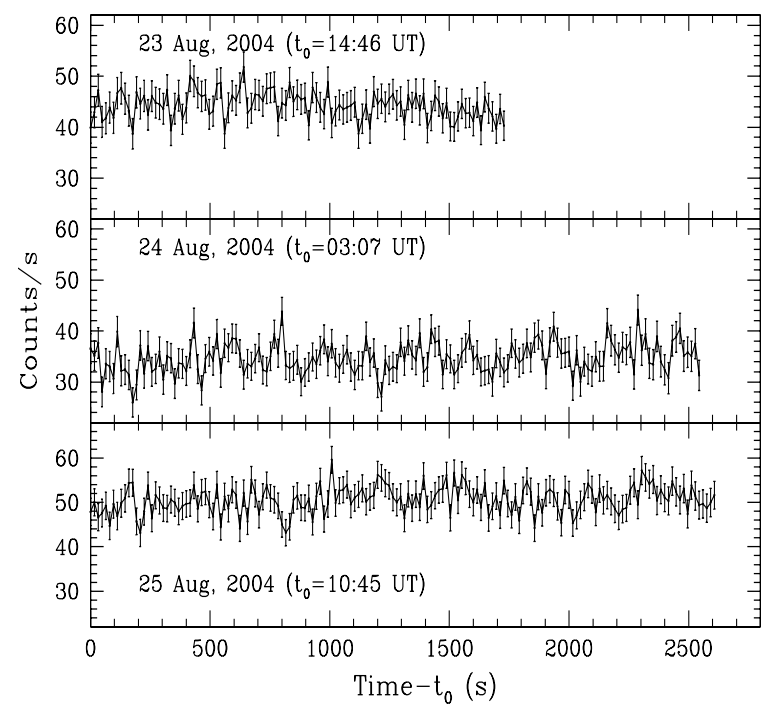

Fig. 2. RXTE lightcurve of 3-25 keV X-ray. On the day of the inferior conjunction (24 August), the count was distinctly lower. The flux in the $3-25 \mathrm{keV}$ region of $2.25 \times 10^{-10} \mathrm{erg} \mathrm{cm}^{-2} \mathrm{~s}^{-1}$ was more than three times lower compared to the flux at superior conjunction (Nandi et al. 2005). Significant short timescale variabilities were observed throughout.

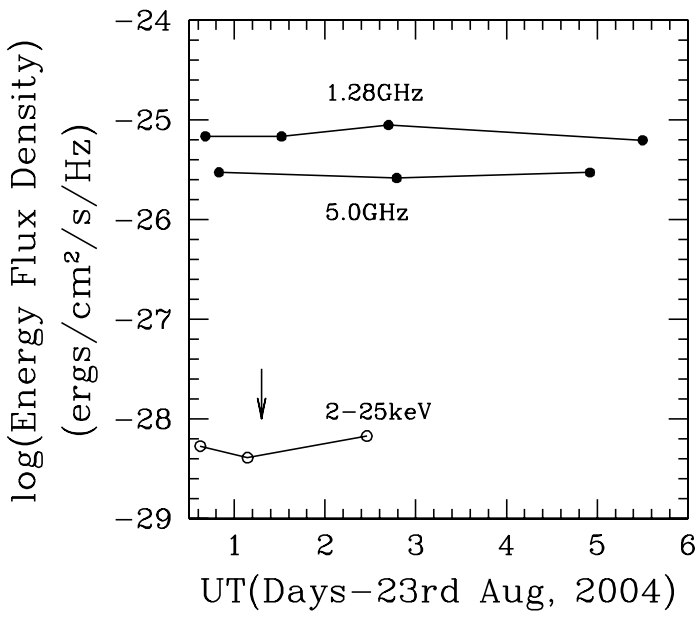

Fig. 3. Variation of the daily average energy flux densities of SS433 in EVN (4.99 GHz), GMRT (1.28 GHz), and RXTE (3-25 keV). The time of the inferior conjunction is marked by an arrow, where X-ray shows a minimum. The apparent "anti-correlation" between the $4.99 \mathrm{GHz}$ and $1.28 \mathrm{GHz}$ data may be due to the blob expansion.

Table 1. Comparison of the fluxes on various dates.

\begin{tabular}{ccccc}
\hline \hline Date & $3-10 \mathrm{keV}$ & Ratio & $10-25 \mathrm{keV}$ & Ratio \\
\hline 13 March 2004 & $3.38 \times 10^{-10}$ & 2.22 & $3.53 \times 10^{-10}$ & 4.77 \\
23 August 2004 & $1.89 \times 10^{-10}$ & 1.24 & $1.08 \times 10^{-10}$ & 1.46 \\
24 August 2004 & $1.52 \times 10^{-10}$ & 1.0 & $0.74 \times 10^{-10}$ & 1.0 \\
25 August 2004 & $2.07 \times 10^{-10}$ & 1.36 & $1.61 \times 10^{-10}$ & 2.18 \\
\hline
\end{tabular}

observation indicates that a significant soft X-ray is emitted from the jet, which is not blocked by the companion. This is expected since it is believed that the companion is an A-type Supergiant with a radius of $40 R_{\odot}$ (Cherepashchuk et al. 2005) and it generally blocks only the hard X-ray emitting region of $\sim 10^{12} \mathrm{~cm}$. Hence, emissions from $z>10^{12} \mathrm{~cm}$ of the jet can reach the observer throughout the precession period.

The spectra of the X-ray data are shown in Fig. 4 for these days and the fitting of the data with bremsstrahlung and two 

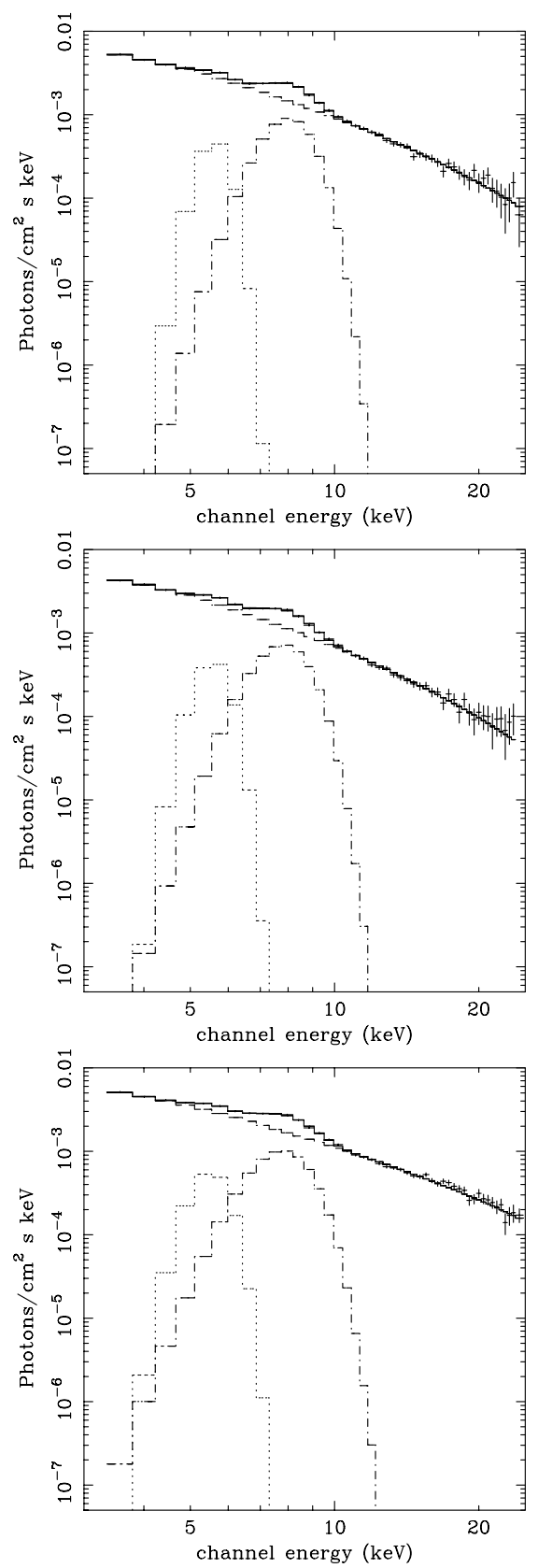

Fig. 4. X-ray spectra in $3-25 \mathrm{keV}$ on 23 (top), 24 (middle), and 25 (bottom) August 2004.

iron lines are also shown. The iron lines correspond to those being emitted from the blue and red-shifted jets. In order to show that only the hard X-rays are preferentially blocked, we show in Table 1 the flux in $3-10 \mathrm{keV}$ and in $10-25 \mathrm{keV}$ in the data of 23, 24, and 25 August 2004. For comparison we also used the RXTE result on the superior conjunction, $\phi=0.5, \psi=0.0$, which is another opportune moment in which all RXTE TOO observation was made at our request. We take the ratio by dividing the flux in each observation by that on the 24th. We find that, while the soft photons on the 24th are reduced by 24 to 36 percent with respect to those in $23 \mathrm{rd}$, the hard photons are reduced by 46 to 118 percent as compared to the 25 th. If we compare the results between superior and inferior conjunctions, the change is even more dramatic: the reduction in the soft photons is by 122 percent, while the reduction in the hard photons is as much as 377 percent.
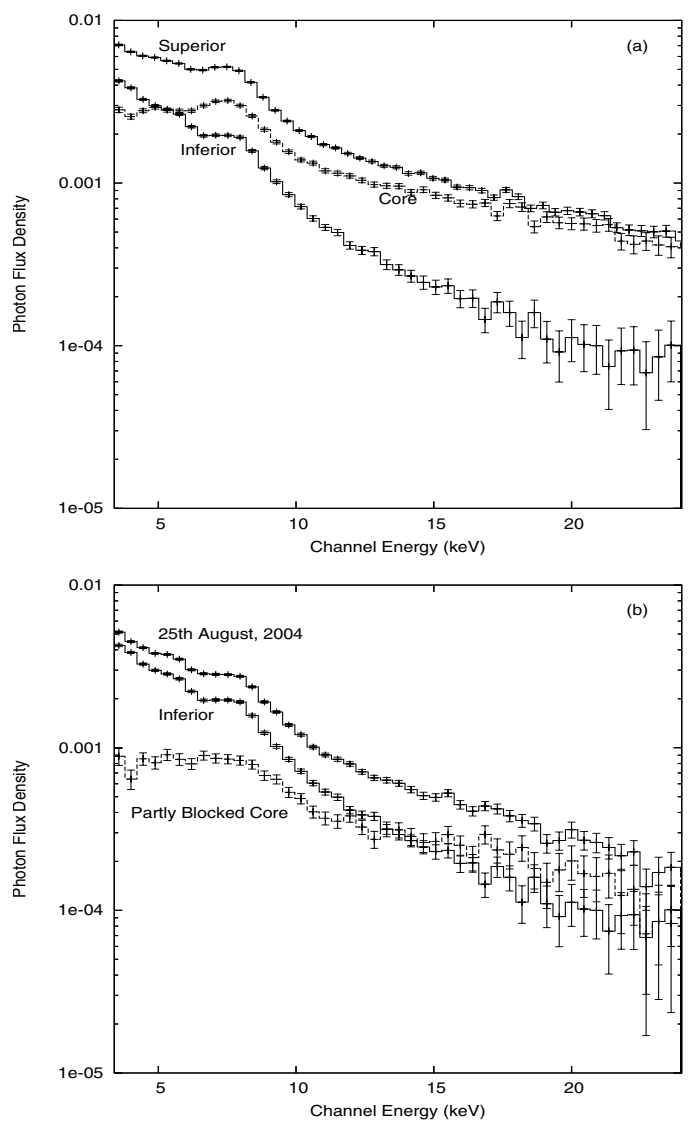

Fig. 5. Photon spectra (solid histograms) of SS433 on a) 13 March 2004 (superior conjunction), and 24 August 2004 (inferior conjunction) and b) 25 August 2004 and 24 August 2004, and the difference between them marked as a) "Core" (dashed histograms) and b) "Partly blocked core". In all the cases, the precessional phase $\psi \sim 0$. The core $(z \lesssim$ $10^{12} \mathrm{~cm}$ ) seems to emit a significant hard X-ray and almost an entire amount of the iron line component as indicated by the bump in a).

Unlike our analysis of the RXTE result, Cherepashchuk et al. (2005) analysed the results using INTEGRAL data and shows that in $25-60 \mathrm{keV}$ range, the reduction at the inferior conjunction as compared to that at the quadrature position $(\phi=0.25)$ is about a factor of 4 (Cherepashchuk et al. 2005). Comparing with Ginga data of about the same period of time, they also show that hard X-rays are preferably blocked during the inferior conjunction.

The bremsstrahlung fits (Fig. 4) give rise to temperature of the emitting regions to be $11.60 \mathrm{keV}$ on the $23 \mathrm{rd}, 9.84 \mathrm{keV}$ on the $24 \mathrm{th}$, and $18.92 \mathrm{keV}$ on the $25 \mathrm{th}$, respectively. This also gives a clear indication that the hard $\mathrm{X}$-rays are coming from the very base of the jet and that the soft X-rays are emerging from a broader region of the jet. This can be seen more clearly in Figs. 5a, b where we plot the photon spectra of the (a) 13 March 2004 (superior conjunction), 24 August 2004 (inferior conjunction), and (b) 25 and 24 August 2004. In both plots, we show the difference (dashed histogram) between these spectra. In (a), the differential spectrum is that of the blocked region of disk and the base of the jet coming from $z \lesssim 10^{12} \mathrm{~cm}$ (including contribution from any accretion shock due to interaction between the accretion stream and the disk), while in (b) the spectrum is due to the difference between the total blockade on the 24th and the partial blockade of the central region on the 25 th. An interesting observation is that most of the contribution from the iron line (the bump at around $7.52 \mathrm{keV}$ that is very close to $7.68 \mathrm{keV}$, the 

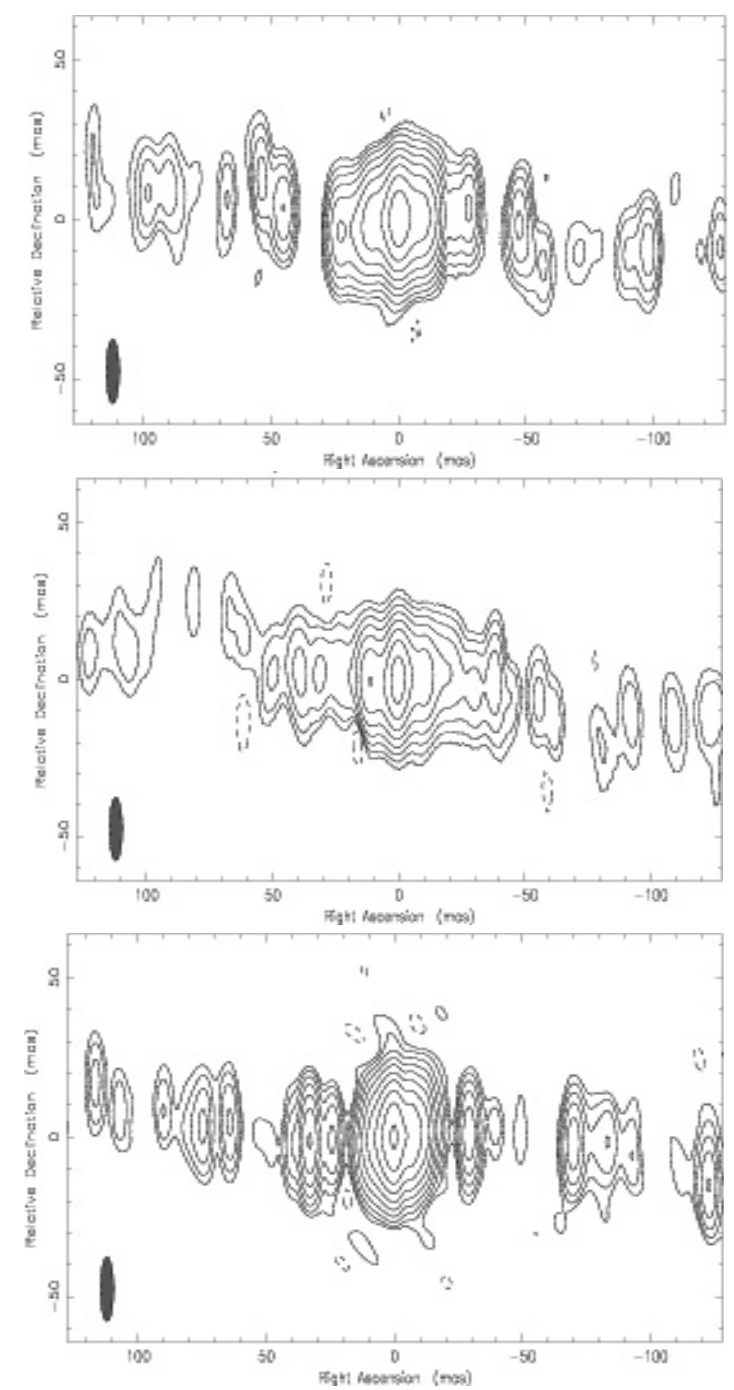

Fig. 6. High resolution VLBI images of SS433 on the 23 (top), 25 (middle), and 27 (bottom) August 2004 at $4.99 \mathrm{GHz}$. Peak brightnesses were 87,75 , and $112 \mathrm{mJy}$ respectively. The images were restored with $20 \times 5$ mas N-S beam. The contour levels increase by a factor of two, the lowest being $0.4 \mathrm{mJy} / \mathrm{beam}$ for 25 August, and $0.2 \mathrm{mJy} / \mathrm{beam}$ at the other two epochs. A blob seen in the blue shifted jet on the 23rd, appears to be separated on the $25 \mathrm{th}$, and the intensity at $4.99 \mathrm{GHz}$ seems to have gone down. At the same time, the intensity at $1.28 \mathrm{GHz}$ was seen to go up indicating an expansion of the blob. See text for details.

expected FeXXVI line at $\phi=0$ ) is also mostly blocked at the inferior conjunction. We note that the core has relatively higher flux of high-energy X-rays. In future, we shall model the inner region using spectra at other precessional and orbital phases.

The evolution of the jet during 23 to 27 August 2004 in $4.99 \mathrm{GHz}$ is shown in Fig. 6. Here high resolution images at $4.99 \mathrm{GHz}$ on 23, 25, and 27 August 2004 are presented. Peak brightnesses were 87,75 , and $112 \mathrm{mJy}$, respectively. The images were restored with a $20 \times 5$ mas $\mathrm{N}-\mathrm{S}$ beam. The contour levels increase with a factor of two, the lowest being $0.4 \mathrm{mJy} / \mathrm{beam}$ for 25 August and $0.2 \mathrm{mJy} / \mathrm{beam}$ at the other two epochs. We note that a blob of matter that was still attached to the core on the 23rd is being detached on the 25th, and totally detached on the 27th. The expansion of the blob on 25 August 2004 may have caused the reduction of the $4.99 \mathrm{GHz}$ flux and enhancement of the $1.28 \mathrm{GHz}$ flux, as is observed in Fig. 3. These $\sim 2$ days of delay can be explained from the model prediction of the flare peak time at different frequencies from $t_{\mathrm{m}, \lambda}=t_{0} \lambda^{\frac{p+4}{4 p+6}}$ (van der Laan 1966).
Here, $p$ is the particle spectral index, $N(E) \propto E^{-p}$, obtained from the energy spectral index $\alpha$ from $p=2 \alpha+1$. Assuming the usual value of $\alpha \sim 0.6$, we have $p \sim 2.2$ and $t_{\mathrm{m}, \lambda}=t_{0} \lambda^{0.42}$. In Pal et al. (2005) we showed that $t_{0}=3$ in a recent flare of SS433. This gives $t_{\mathrm{m}, 23 \mathrm{~cm}}-t_{\mathrm{m}, 6 \mathrm{~cm}} \sim 4.83$ days. Thus, it seems that the blob was at its peak at $4.99 \mathrm{GHz}$ on or around 20 August 2004, long before the inferior conjunction. It is then steadily decreasing until the 25 th, while the intensity at $1.28 \mathrm{GHz}$ is steadily increasing, subsequently peaking on the 25 th.

\section{Discussion and concluding remarks}

In this paper, we have presented a recent multi-wavelength observation of the enigmatic compact object SS433 at a very rare opportunity, when the blue jet was pointing directly towards us $(\psi=0)$, while the companion was totally blocking the central object and the base of the jet (inferior conjunction, $\phi=0$ ). We saw an X-ray minimum at this conjunction, and upon comparison with the observation at the superior conjunction (with $\phi=0.5)$ as well as a day before and after the inferior conjunction, we found that a significant fraction of the hard X-ray $(>10 \mathrm{keV})$ was blocked by the companion, while only a small fraction of the soft X-ray was blocked. This shows that most of the hard X-ray was coming less than $10^{11-12} \mathrm{~cm}$ from the centre, while the soft X-rays were emitted from regions of the jet located farther out. We also found the spectrum of the blocked region and a strong bump at the blue-shifted FeXXVI line, indicating that most of the iron emission is also from the central region. Not surprisingly, the radio emission showed no correlation with the conjunction itself. However, we clearly identified a blob separation in our VLBI data, which is estimated to have taken place at around 20 August 2005 and found that the intensity at $1.28 \mathrm{GHz}$ was monotonically increasing, while that at $4.99 \mathrm{GHz}$ was monotonically decreasing.

Acknowledgements. This work was partly supported by a CSIR SRF (S.P.) grant. The work of A.N. is supported by a DST project. The authors thank the EVN team, especially Dr. Z. Paragi, for giving us the observing time. The European VLBI Network is a joint facility of European, Chinese, South African, and other radio-astronomy institutes funded by their national research councils.

\section{References}

Band, D. L., \& Gordon, M. A. 1989, ApJ, 338, 945

Chakrabarti, S. K., Pal, S., Nandi, A., Anandarao, B. G., \& Mondal, S. 2003, ApJ, 595, L45

Chakrabarti, S. K., Anandarao, B. G., Pal, S., et al. 2005, MNRAS, 362, 957

Cherepashchuk, A. M., Sungaex, R. A., Fabrika, S. N., et al. 2005, A\&A, 437, 561

Ciatti, F., Mammano, A., Bartolini, C., et al. 1981, 95, 177

Diamond, P. J. 1995, in Very Long Baseline Interferometry and the VLBA, ed. J. A. Zensus, P. J. Diamond, \& P. J. Napier, ASP Conf. Ser., 82, 227

Kotani, T., Band, D., Cherepashchuk, A. M., et al. 1999, Astron. Nachr., 320, 335

Nandi, A., Chakrabarti, S. K., Belloni, T., \& Goldoni, P. 2005, MNRAS, 359, 629

Neizvestnyj, S. I., Pustilnik, S. A., \& Efremov, V. G. 1980, Sov. Astron. Lett., 6, 368

Pal, S., Chakrabarti, S. K., Kraus, A., \& Mandal, S. 2005, Bull. Astron. Soc. India, in press

Paragi, Z., et al. 2005, in Triggering Relativistic Jets, Rev. Mex. Astron. Astrofis., $\mathrm{CD}$ version, in press

Revnivtsev, M., Burenin, R., Fabrika, S., et al. 2004, A\&A, 424, L5

Seaquist, E. R., Gilmore, W. S., Johnston, K. J., \& Grindlay, J. E. 1982, ApJ, 260,220

Shepherd, M. C. 1997, in ed. G. Hunt, \& H. E. Payne, Astronomical Data Analysis Software and Systems VI, ASP Conf. Ser., 125, 77

van der Laan, H. 1966, Nature, 211, 1131

Vermeulen, R. C., McAdam, W. B., Trushkin, S. A., et al. 1993, A\&A, 270, 189 